\title{
Characterisation of the clinical phenotype in Phelan-McDermid syndrome
}

\author{
Mónica Burdeus-Olavarrieta 1,2,3* D, Antonia San José-Cáceres ${ }^{1,2}$, Alicia García-Alcón ${ }^{1,2,4}$, Javier González-Peñas ${ }^{1,2,5}$, \\ Patricia Hernández-Jusdado ${ }^{1}$ and Mara Parellada-Redondo ${ }^{1,2,4}$
}

\begin{abstract}
Background: Phelan-McDermid syndrome (PMS) is a rare genetic disorder compromising the $22 q 13$ terminal region and affecting SHANK3, a gene crucial to the neurobehavioural phenotype and strongly linked to autism (ASD) and intellectual disability (ID). The condition is characterised by global developmental delay, ID, speech impairments, hypotonia and autistic behaviours, although its presentation and symptom severity vary widely. In this study, we provide a thorough description of the behavioural profile in PMS and explore differences related to deletion size and language ability.

Methods: We used standard clinical assessment instruments to measure altered behaviour, adaptive skills and autistic symptomatology in sixty participants with PMS (30 females, median age 8.5 years, SD=7.1). We recorded background information and other clinical manifestations and explored associations with deletion size. We performed descriptive and inferential analyses for group comparison.

Results: We found delayed gross and fine motor development, delayed and impaired language ( 70\% of participants non or minimally verbal), ID of different degrees and adaptive functioning ranging from severe to borderline impairment. Approximately $40 \%$ of participants experienced developmental regression, and half of those regained skills. Autistic symptoms were frequent and variable in severity, with a median ADOS-2 CSS score of 6 for every domain. Sensory processing anomalies, hyperactivity, attentional problems and medical comorbidities were commonplace. The degree of language and motor development appeared to be associated with deletion size.

Conclusions: This study adds to previous research on the clinical descriptions of PMS and supports results suggesting wide variability of symptom severity and its association with deletion size. It makes the case for suitable psychotherapeutic and pharmacological approaches, for longitudinal studies to strengthen our understanding of possible clinical courses and for more precise genomic analysis.
\end{abstract}

Keywords: Phelan-McDermid syndrome, 22q13 deletion syndrome, SHANK3, Intellectual disability, Autism

\section{Background}

Phelan-McDermid syndrome (22q13 deletion syndrome or PMS), exhaustively explored by Phelan and McDermid [1], is a rare genetic disorder characterised by global

\footnotetext{
* Correspondence: monica.burdeus@iisgm.com

'Department of Child and Adolescent Psychiatry, Institute of Psychiatry and Mental Health, Hospital General Universitario Gregorio Marañón, Calle Ibiza 43, 28009 Madrid, Spain

${ }^{2}$ liSGM, Instituto de Investigación Sanitaria Gregorio Marañón, Madrid, Spain Full list of author information is available at the end of the article
}

developmental delay, hypotonia, intellectual disability (ID), severe speech impairments and autism spectrum disorder (ASD) to a variable degree [2]. Other manifestations include minor dysmorphic features and somatic conditions, especially gastrointestinal reflux and renal problems $[3,4]$. Sometimes additional neurological problems occur, including seizures, reduced pain and temperature sensitivity or structural and functional brain abnormalities [3, 5]. Researchers also report a high

(c) The Author(s). 2021 Open Access This article is licensed under a Creative Commons Attribution 4.0 International License, which permits use, sharing, adaptation, distribution and reproduction in any medium or format, as long as you give appropriate credit to the original author(s) and the source, provide a link to the Creative Commons licence, and indicate if changes were made. The images or other third party material in this article are included in the article's Creative Commons licence, unless indicated otherwise in a credit line to the material. If material is not included in the article's Creative Commons licence and your intended use is not permitted by statutory regulation or exceeds the permitted use, you will need to obtain permission directly from the copyright holder. To view a copy of this licence, visit http://creativecommons.org/licenses/by/4.0/ The Creative Commons Public Domain Dedication waiver (http://creativecommons.org/publicdomain/zero/1.0/) applies to the data made available in this article, unless otherwise stated in a credit line to the data. 
prevalence of psychiatric comorbidities and developmental regression $[6,7]$. The exact prevalence of the syndrome remains unknown, and the condition is likely underdiagnosed, due to its unspecific clinical phenotype and the need for molecular genetic testing to establish a diagnosis [8]. Recent descriptions of this syndrome reveal several clinical features, such as ASD and ID, attention deficit, hyperactivity and impulsivity, aggressive behaviour, language impairments and medical comorbidities $[4,9]$.

The association between the SHANK gene family and neurodevelopmental disorders is well established [1012]. Some researchers consider the loss of a functional copy of the SHANK3 gene, which is responsible for the codification of a scaffolding protein in the postsynaptic density, the cause of the main clinical features of PMS $[3,13]$. Recent studies suggest that deletions or mutations compromising SHANK3 can cause a monogenic form of both ASD and ID, accounting for around 1\% of the overall ASD cases $[8,14]$ and $0.3-1 \%$ of the ID $[15$, 16]. Researchers have explored genotype-phenotype associations, showing that deletion size and locus are often correlated with speech abilities [3, 17], hypotonia and dysmorphic features [13, 18] and adaptive skills $[9,19]$. Findings concerning other traits, such as autistic or aggressive behaviour, are inconsistent $[14,17,20]$. In this study, we aim to conduct a deep phenotypic characterisation of the clinical and neurobehavioural traits of a large national sample of participants with PhelanMcDermid syndrome, using standardised, robust instruments to assess repetitive or otherwise altered behaviours, hyperactivity, attentional problems, sensory processing difficulties, global adaptive functioning and autistic features. Specifically, we want to explore the similarity of the behavioural profiles between verbal and non-verbal individuals. Therefore, our research questions read: \#1 Are the characteristics of our PMS sample like other cohorts described? \#2 How is language ability related to other phenotypic characteristics? Do the clinical profiles of verbal and non-verbal individuals differ?

We expect our findings to be in accordance with those previously reported in the aforementioned clinical descriptions, including developmental and language delay, presence of ASD traits and challenging behaviours (e.g. aggression, hyperactivity), as well as certain correlations between these characteristics and the size of the deletion.

\section{Methods}

\section{Participants}

All individuals from the Spanish Phelan-McDermid Association (89 families) and also patients with an identified SHANK3 alteration (1 family) assisted in the regional AMITEA programme (Comprehensive Medical
Care-Autism Spectrum Disorders) [21] of the Hospital General Universitario Gregorio Marañón in Madrid through the end of 2018 were invited to participate. Sixty children and adults (50\% female), between 11 months and 41 years of age $(M=10.6$ years, median $=8.5$, $\mathrm{SD}=7.1$ ), participated (response rate $=66.7 \%$ ). Sample characteristics are in Table 1.

The only inclusion requirement was having a diagnosis of Phelan-McDermid syndrome. Trained psychologists and psychiatrists conducted the assessments at Gregorio Marañón hospital. Caregivers gave informed consent. The hospital ethics committee approved the study.

\section{Materials and assessment}

- Autistic symptomatology was quantified with the following instruments: Autism Diagnostic Observation Schedule-2 (ADOS-2 [22]), Autism Diagnostic Interview-Revised (ADI-R [23]), Repetitive Behaviours Scale-Revised (RBS-R [24];), Social Responsiveness Scale (SRS [25];) and Sensory Profile-2 (SP-2 [26];). ADOS-2 is a semi-structured, standardised assessment of communication, social interaction, play and restricted and repetitive behaviours; we calculated ADOS-2 calibrated severity scores (CSS) [27] to offer a comparable measure of ASD severity across ages and modules. ADI- $\mathrm{R}$ is an extensive interview conducted with caregivers of individuals suspected of having ASD, which provides a thorough assessment of early development in three domains: reciprocal social interactions (A); language/communication (B); and restricted, repetitive and stereotyped behaviours and interests $(\mathrm{C})$. Both ADOS-2 and ADI-R are gold-standard instruments for the diagnosis of ASD and in combination have proven to offer a reliable assessment of autistic symptomatology; however, their validity in samples with severe to profound ID is decreased [28]. In this work, ADOS-2 and ADI-R scores are used to quantify autistic symptoms, and no clinical diagnosis of autism is intended. ADI-R items $11-28$, which define regression as a loss of consistently acquired skills during at least 3 months, provided information about regression. Parents completed the parentrated RBS-R, which assesses repetitive behaviours and the parent-rated SRS, which measures the severity of autistic symptoms in natural settings for verbal individuals, along with the SP-2 to assess children's sensory processing systems and patterns.

- Adaptive behaviour was evaluated by means of the Vineland Adaptive Behavior Scales-3 (VABS-3) in its comprehensive parent/caregiver form [29]. This form was used and not the interview due to time constraints. These scales have been a leading 
Table 1 Main sociodemographic and clinical characteristics of the sample

\begin{tabular}{|c|c|c|}
\hline Sample characteristics & $\mathrm{N}$ & $\%$ \\
\hline \multicolumn{3}{|l|}{ Sex } \\
\hline Male | Female & $30 \mid 30$ & $50 \mid 50 \%$ \\
\hline \multicolumn{3}{|l|}{ Age } \\
\hline $0-5$ years & 14 & $23.3 \%$ \\
\hline $6-11$ years & 25 & $41.7 \%$ \\
\hline $12-17$ years & 12 & $20.0 \%$ \\
\hline$\geq 18$ years & 9 & $15.0 \%$ \\
\hline \multicolumn{3}{|l|}{ Socioeconomic status } \\
\hline Low | M-low | Middle & $1|14| 9$ & $1.6 \%|23.3 \%| 15 \%$ \\
\hline M-upper | Upper & 17 | 19 & $28.3 \% \mid 31.6 \%$ \\
\hline \multicolumn{3}{|l|}{ Gestational age } \\
\hline Pre-term (<37 weeks) & 12 & $20.0 \%$ \\
\hline Full-term (38-42 weeks) & 48 & $80.0 \%$ \\
\hline Language $(\geq 3$ yo) & 54 & \\
\hline No words & 28 & $51.9 \%$ \\
\hline Single words & 7 & $13.0 \%$ \\
\hline Simple sentences & 11 & $20.4 \%$ \\
\hline Fluid speech & 8 & $14.8 \%$ \\
\hline \multicolumn{3}{|l|}{ Developmental regression/Loss of skills (parent reported) } \\
\hline Yes | No & $25 \mid 35$ & 41.7 | 58.3\% \\
\hline \multicolumn{3}{|l|}{ Recovered skills } \\
\hline Yes | No & $12 \mid 13$ & 48 | 52\% \\
\hline Autistic and other clinically relevant behaviours & $\mathbf{N}$ & $\%$ \\
\hline ADOS-2* & 48 & - \\
\hline Total-CSS 1-3 & 12 & $25.0 \%$ \\
\hline Total-CSS 4-5 & 8 & $16.7 \%$ \\
\hline Total-CSS 6-10 & 28 & $58.3 \%$ \\
\hline $\mathrm{CBCL}^{*}(1.5-5$ years $\mid 6-18$ years $)$ & $10 \mid 28$ & \\
\hline Emotionally reactive & $4 \mid \mathrm{n} / \mathrm{a}$ & $40 \%$ |n/a \\
\hline Anxious/depressed & $0 \mid 0$ & $0 \% \mid 0 \%$ \\
\hline Somatic complaints & $0 \mid 6$ & $0 \% \mid 21 \%$ \\
\hline Sleep problems & $1 \mid \mathrm{n} / \mathrm{a}$ & $10 \% \mid n / a$ \\
\hline Attention problems & $6 \mid 20$ & $60 \% \mid 71 \%$ \\
\hline Withdrawn & $7 \mid 7$ & $70 \%$ | 25\% \\
\hline Aggressive & $3 \mid 6$ & $30 \% \mid 21 \%$ \\
\hline Rule-breaking & $\mathrm{n} / \mathrm{a} \mid 3$ & n/a | 11\% \\
\hline Social problems & $\mathrm{n} / \mathrm{a} \mid 18$ & n/a | 64\% \\
\hline Thought problems & $\mathrm{n} / \mathrm{a} \mid 15$ & n/a | 54\% \\
\hline $\mathrm{SDQ}^{*}$ & 33 & \\
\hline Emotional symptoms & 1 & $3.0 \%$ \\
\hline Conduct problems & 2 & $6.1 \%$ \\
\hline Hyperactivity & 21 & $63.6 \%$ \\
\hline Peer relations difficulties & 22 & $66.7 \%$ \\
\hline
\end{tabular}


Table 1 Main sociodemographic and clinical characteristics of the sample (Continued)

\begin{tabular}{lll}
\hline Sample characteristics & $\mathbf{N}$ & $\%$ \\
\hline Difficulties with prosocial behaviour & 28 & $84.8 \%$ \\
Total & 17 & $51.5 \%$ \\
\hline
\end{tabular}

*Language: We used 3 years as the developmental threshold for language acquisition, and only considered participants over this age for descriptive purposes to better distinguish delay in acquisition from absence of speech. The remaining 6, however, were minimally verbal. *ADOS-2 (Autism Diagnostic Observation Scale2): Calibrated severity scores indicate severity of autistic symptoms. ${ }^{*} \mathrm{CBCL}$ (Child Behavior Checklist): One version for children aged 1.5 to 5 years and another for children aged 6 to 18 with five shared subscales; values indicate individuals who scored over the cutoff for borderline clinical relevance. *SDQ (Strengths and Difficulties Questionnaire): Values indicate individuals who scored over the cutoff for borderline clinical relevance

instrument for supporting the diagnosis of intellectual disabilities for the last decades, providing scores for communication, daily living skills, socialization and motor skills domains, as well as an overall adaptive behaviour composite. Motor domain only considers children younger than 10 years. The VABS-3 has a mean standard score of 100 and SD of 15 for domains and a mean v-score of 15 and SD of 3 for subdomains.

- Other behavioural difficulties were assessed through the following: (i) Aberrant Behavior Checklist-Community (ABC-C [30]), a checklist for assessing problem behaviours of children and adults with developmental disabilities; (ii) Child Behavior Checklist (CBCL [31]), a report for identifying problem behaviours in children aged 1.5-5 and 618 years; and (iii) The Strengths and Difficulties Questionnaire (SDQ [32]), a brief screening questionnaire for children aged 4-17. Both the CBCL and SDQ offer cutoff scores for clinically relevant and borderline altered behaviours. To account for all altered behaviour, we considered scores above the borderline cutoff.

- Background information regarding early development, medical comorbidities, previous neurophysiological, imaging procedures, etc. was also gathered using a self-devised interview with parents. We used the Cumulative Illness Rating Scale (CIRS) [33] in a retrospective manner to obtain information on medical events over participants' lifetimes.

- Genetic information was provided by parents and caregivers through existing clinical genetic reports. We only used deletion size. Genetic tests leading to diagnosis included different assessments such as Karyotype, FISH, MLPA and array-CGH.

\section{Data analysis and cluster analysis}

Study data were stored and managed using REDCap (Research Electronic Data Capture) electronic data capture tools hosted at Instituto de Investigación Sanitaria Gregorio Marañón. REDCap is a secure, web-based application designed to support data capture for research studies [34].
We performed descriptive and inferential analyses, including unpaired T-tests and one-way ANOVA for group comparisons of continuous variables, Chi-square tests to compare proportions and Pearson's correlation coefficients to explore associations between behavioural variables and deletion size. For those variables with nonnormal distributions, we performed non-parametric statistical tests, including Mann-Whitney U tests for group comparisons of continuous variables, Wilcoxon signedrank test for paired comparisons and Spearman rank correlation coefficients. We computed 95\% CI with bootstrapping ( $\mathrm{BCa} 95 \% \mathrm{CI}$ ) for the parameter estimates and reported the $95 \% \mathrm{CI}$ of mean differences for parametric tests. We used PASW Statistics 18 [35] for statistical analysis and R-3.6.3 [36] to generate graphs.

Ward's hierarchical cluster analysis was performed on 42 individuals using the following variables: deletion size, sex, developmental regression, language status and ADOS-2 total CSS score to explore grouping of clinical variability.

\section{Results}

\section{Sample description}

Participants $(\mathrm{n}=60)$ were predominantly younger than 18 years (age ranging from 0.92 to 23 years, one individual aged 41 years; median $=8.54, \mathrm{SD}=7.1$ ), and sex distribution was even. Sample characteristics are summarised in Table 1.

Forty-eight participants had ADOS-2 scores: 46 administered during this study, 1 administered 2 years earlier by a research-trained professional in AMITEA (same module) and 1 from a private report from the previous year. The unassessed cases $(n=12)$ either showed important mobility limitations and severe ID (estimated $\mathrm{MA}<15 \mathrm{mo}$ ) or lived too far away to visit. Module 1 was used for 35 participants, module 2 for 7 , module 3 for 5 and module $\mathrm{T}$ for 1 .

\section{Clinical findings \\ Early development}

Average age at independent walking was 22 months $(\mathrm{SD}=8.2$, range $=[11-42]) ; 4$ participants older than 42 months $(7.7 \%)$ could not walk at the time of the assessment. Thirty-two participants showed delayed walking, defined as walking unaided after 18 months of age, and 
at least 21 had not crawled. Overall, 54 (90\%) had delayed fine and/or gross motor development.

Considering only participants aged 3 and up, 28 (51.9\%) were non-verbal, 7 (13.0\%) used single words, 11 (20.4\%) used simple sentences and 8 (14.8\%) used complex sentences or fluid speech. Age at acquisition of language ranged from 8 months to 6.5 years for words and from 2.5 to 14 years for sentences. On average, participants produced their first words at $\sim 32$ months and first sentences at $\sim 6$ years. For descriptive purposes, we divided the sample in two groups, "Non-verbal or minimally verbal", used interchangeably for subjects producing none to single words and "Verbal" for those who use sentences.

\section{Developmental regression}

Parents reported a history of developmental regression in 24 cases and an additional case of "loss of skills". Of these, only 6 met the strict ADI-R criteria for language regression. However, in 17 cases, parents reported a noticeable loss of skills in speech production (i.e. abruptly stopped babbling or using acquired words), regardless of initial language level, and 21 reported regression or loss of skills in other domains: social engagement and reciprocity (21), motor skills (6), self-help skills (6) and play (3). In at least 4 cases, regression was possibly related to medical causes (mononucleosis, severe bronchitis, severe bronchospasms, metabolic acidosis).

Fourteen participants experienced regression before or at 30 months (mean $=48.7$, range $=[11-168]$ months). Notably, 4 cases occurred at age 10 and older: One involved regression in speech production with regained abilities; another was related to language articulation; the remaining 2 experienced a generalised regression with emotional dysregulation at ages 13 and 14 .

Of the 25 cases, ten individuals regained a similar level of functioning after times ranging from 5 months to 9 years and 2 regained some of the abilities ( 6 out of 7 verbal participants and 6 out of 18 non-verbal participants). Thirteen participants (22\%) experienced a persistent loss of skills. We explored possible associations between regression and sex $\left(X^{2}=.069, p=.793, \mathrm{~V}=.034\right)$, language level $\left(X^{2}=.396, p=.529, \mathrm{~V}=.087\right.$, age $\geq 3$ years old $)$ and deletion size $(t(51)=1.324, p=.191$, Hedge's $g=0.369)$.

\section{Autism profile}

For the whole sample, median ADOS-2 CSS was 6 for SA, RRB and total score. Table 2 shows ASD symptomatology separated by language. ADOS-2-CSS and ADI-R $A$ and $C$ mean scores were similar or slightly higher for minimally verbal individuals, although with considerable CI overlap.

\section{Social affect and social interaction}

Social affect scores as measured by the ADOS-2 SA-CSS were similar for verbal and non-verbal participants but seemed to be higher (although with CI overlap) for nonverbal individuals in the ADI-R domain A: reciprocal social interaction (see Table 2).

\section{Repetitive and restrictive behaviours}

Scores in the ADOS-2 RRB-CSS and the ADI-R domain $\mathrm{C}$ were similar for verbal and non-verbal participants (see Table 2). Within the RBS-R questionnaire, verbal individuals appeared to score higher than non-verbal in the ritualistic subscale $(U=111.500, \mathrm{Z}=-2.614, p=.009$, $\eta^{2}=0.159$ ).

\section{Sensory processing difficulties}

Scores from the SP-2 separated by language level can be found in Table 2. Considering all participants, at least $60 \%$ scored over percentile 80 in the sensory systems "Touch", "Movement" and "Body position", accounting for sensory processing difficulties such as increased reactivity to tactile stimuli, search of abrupt vestibular input, low muscle tone and increased pain/temperature tolerance $-45 \%$ reported a high pain threshold. Other areas, such as oral or visual processing, were not particularly affected. With regards to sensory patterns, over $80 \%$ showed impairments in registration (i.e. ignoring or failing to perceive certain stimuli, e.g. seeming disconnected from the environment, having a high pain threshold or losing eye contact in sustained interaction), and over $60 \%$ reported attentional problems, such as poor attention span and being easily distracted. Detailed information is presented in Supplementary table 1.

\section{Adaptive functioning}

We obtained results for 46 participants (all ages considered; characteristics of participants missing VABS were similar to completers). Mean (SD) scores from the VABS-3 domains were communication 39.28 (18.62), daily living skills 49.15 (17.90), socialization 51.37 (19.72) and total adaptive composite 49.87 (16.94). Vscale scores and age equivalents (AE) were largely below normative values (medians reported): receptive 4 (AE $16.50 \mathrm{mo})$, expressive $2(\mathrm{AE} 9.50 \mathrm{mo})$, written $1(\mathrm{n} / \mathrm{a})$, personal 4.5 (AE 23mo), domestic 7 (n/a), community 3.5 $(\mathrm{n} / \mathrm{a})$, interpersonal $6(\mathrm{AE} 15.50 \mathrm{mo})$, play $6(\mathrm{AE} 15.50)$, coping $6(\mathrm{n} / \mathrm{a})$, gross motor 8 (AE 20mo) and fine motor 5 (AE 17mo). Regarding language, $\mathrm{V}$-scale scores for receptive and expressive subdomains fell within the normative ranges for 5 and 3 participants, respectively. To further look at these communicative skills, we explored possible intra-subject differences between scores in receptive and expressive subdomains $(Z=-1.100, p=.271$, Wilcoxon's test). Results showing receptive and 
Table 2 ASD symptomatology by language level

\begin{tabular}{|c|c|c|c|c|c|c|}
\hline & & \multicolumn{2}{|c|}{ Verbal } & \multicolumn{2}{|c|}{ Minimally verbal ( $\geq 3$ yo) } & \multirow[b]{2}{*}{ Comparison } \\
\hline & & $n$ & Mean (SD) [range] & $n$ & Mean (SD) [range] & \\
\hline \multirow[t]{3}{*}{ ADOS-2 } & SA-CSS & 18 & $\begin{array}{l}5.50(2.33)[1-10] \\
\text { BCa } 95 \% \mathrm{Cl}[4.44,6.61]\end{array}$ & 27 & $\begin{array}{l}5.56(2.78)[2-10] \\
\text { BCa } 95 \% \mathrm{Cl}[4.32,6.71]\end{array}$ & $U=229.500, Z=-.316, p=.752, \eta^{2}=0.002$ \\
\hline & RRB-CSS & & $\begin{array}{l}5.11(2.59)[1-10] \\
\text { BCa } 95 \% \mathrm{Cl}[4.00,6.22]\end{array}$ & & $\begin{array}{l}6.22(1.48)[1-9] \\
\text { BCa } 95 \% \mathrm{Cl}[5.58,6.80]\end{array}$ & $U=174.000, Z=-1.656, p=.098, \eta^{2}=0.062$ \\
\hline & Total-CSS & & $\begin{array}{l}5.00(2.17)[1-9] \\
\text { BCa } 95 \% \mathrm{Cl}[3.89,6.09]\end{array}$ & & $\begin{array}{l}5.22(2.31)[2-10] \\
\text { BCa } 95 \% \mathrm{Cl}[4.32,6.15]\end{array}$ & $U=230.500, Z=-.297, p=.766, \eta^{2}=0.002$ \\
\hline \multirow[t]{3}{*}{ ADI-R } & A & 18 & $\begin{array}{l}16.33(6.71)[6-28] \\
\text { BCa } 95 \% \text { Cl }[13.54,19.24]\end{array}$ & 32 & $\begin{array}{l}20.94(5.84)[6-30] \\
\text { BCa } 95 \% \mathrm{Cl}[18.91,22.91]\end{array}$ & $U=173.500, Z=-2.320, p=.020, \eta^{2}=0.108$ \\
\hline & B & & $\begin{array}{l}13.44(5.68)[2-23] \\
\text { BCa } 95 \% \text { Cl }[10.85,16.06]\end{array}$ & & $\begin{array}{l}12.25(3.06)[0-14] \\
\text { BCa } 95 \% \mathrm{Cl}[11.01,13.19]\end{array}$ & $\mathrm{n} / \mathrm{a}$ \\
\hline & C & & $\begin{array}{l}4.44(2.64)[0-9] \\
\text { BCa } 95 \% \mathrm{Cl}[3.33,5.45]\end{array}$ & & $\begin{array}{l}4.62(2.34)[0-8] \\
\text { BCa } 95 \% \mathrm{Cl}[3.84,5.49]\end{array}$ & $t(48)=.250,95 \% \mathrm{Cl}[-1.27,1.63], p=.803$ \\
\hline \multirow[t]{6}{*}{ SRS } & Total & 11 & $\begin{array}{l}87.27(35.00)[26-141] \\
\text { BCa } 95 \% \text { Cl }[66.76,106.88]\end{array}$ & $\mathrm{n} / \mathrm{a}$ & & \\
\hline & Social Awareness & & $\begin{array}{l}10.82(4.26)[4-19] \\
\text { BCa } 95 \% \text { Cl }[8.73,13.00]\end{array}$ & & & \\
\hline & Social Cognition & & $\begin{array}{l}18.36(6.07)[9-26] \\
\text { BCa } 95 \% \mathrm{Cl}[14.79,21.82]\end{array}$ & & & \\
\hline & Social Communication & & $\begin{array}{l}28.00(13.02)[4-46] \\
\text { BCa } 95 \% \text { Cl }[19.69,34.95]\end{array}$ & & & \\
\hline & Social Motivation & & $\begin{array}{l}13.91(7.11)[4-28] \\
\text { BCa } 95 \% \mathrm{Cl}[10.18,17.62]\end{array}$ & & & \\
\hline & Autistic Mannerisms & & $\begin{array}{l}15.27(7.59)[4-25] \\
\text { BCa } 95 \% \text { Cl }[10.73,19.73]\end{array}$ & & & \\
\hline \multirow[t]{6}{*}{ RBS-R } & Stereotyped & 15 & $\begin{array}{l}1.67(2.47)[0-8] \\
\text { BCa } 95 \% \text { Cl }[.60,2.80]\end{array}$ & 28 & $\begin{array}{l}3.32(3.35)[0-12] \\
\text { BCa } 95 \% \mathrm{Cl}[2.19,4.67]\end{array}$ & $U=141.500, Z=-1.790, p=.073, \eta^{2}=0.075$ \\
\hline & Self-injurious & & $\begin{array}{l}1.60(1.59)[0-4] \\
\text { BCa } 95 \% \mathrm{Cl}[.87,2.27]\end{array}$ & & $\begin{array}{l}1.89(2.20)[0-8] \\
\text { BCa } 95 \% \mathrm{Cl}[1.21,2.69]\end{array}$ & $U=208.000, Z=-.053, p=.958, \eta^{2}<0.001$ \\
\hline & Compulsive & & $\begin{array}{l}4.33(6.62)[0-20] \\
\text { BCa } 95 \% \mathrm{Cl}[1.69,7.27]\end{array}$ & & $\begin{array}{l}2.14(2.92)[0-10] \\
\text { BCa } 95 \% \mathrm{Cl}[1.17,3.40]\end{array}$ & $U=180.000, Z=-.792, p=.429, \eta^{2}=0.015$ \\
\hline & Ritualistic & & $\begin{array}{l}4.33(4.34)[0-14] \\
\text { BCa } 95 \% \mathrm{Cl}[2.41,6.45]\end{array}$ & & $\begin{array}{l}1.54(2.22)[0-7] \\
\text { BCa } 95 \% \text { Cl }[.77,2.48]\end{array}$ & $U=111.500, Z=-2.614, p=.009, \eta^{2}=0.159$ \\
\hline & Sameness & & $\begin{array}{l}6.53(7.61)[0-27] \\
\text { BCa } 95 \% \text { Cl }[3.45,10.22]\end{array}$ & & $\begin{array}{l}3.46(4.46)[0-13] \\
\text { BCa } 95 \% \mathrm{Cl}[1.72,5.39]\end{array}$ & $U=155.000, Z=-1.435, p=.151, \eta^{2}=0.048$ \\
\hline & Restricted & & $\begin{array}{l}1.73(3.33)[0-12] \\
\text { BCa } 95 \% \text { Cl }[.47,3.27]\end{array}$ & & $\begin{array}{l}1.68(2.31)[0-8] \\
\text { BCa } 95 \% \text { Cl }[.96,2.55]\end{array}$ & $U=187.000, Z=-.632, p=.527, \eta^{2}=0.009$ \\
\hline \multirow[t]{8}{*}{ SP-2 } & Auditory & 10 & $\begin{array}{l}18.90(5.63)[13-31] \\
\text { BCa } 95 \% \text { Cl }[15.90,22.56]\end{array}$ & 22 & $\begin{array}{l}19.45(5.57)[9-30] \\
\text { BCa } 95 \% \mathrm{Cl}[17.23,21.73]\end{array}$ & $t(30)=.260,95 \% \mathrm{Cl}[-3.80,4.91], p=.796$ \\
\hline & Visual & & $\begin{array}{l}11.80(2.57)[7-15] \\
\text { BCa } 95 \% \mathrm{Cl}[10.30,13.30]\end{array}$ & & $\begin{array}{l}11.50(4.70)[4-22] \\
\text { BCa } 95 \% \text { Cl }[9.59,13.42]\end{array}$ & $U=89.500, Z=-.838, p=.411, \eta^{2}=0.022$ \\
\hline & Touch & & $\begin{array}{l}25.40 \text { (5.76) [16-36] } \\
\text { BCa 95\% Cl [22.10, 28.60] }\end{array}$ & & $\begin{array}{l}24.82(7.80)[14-42] \\
\text { BCa } 95 \% \text { Cl }[21.75,28.18]\end{array}$ & $t(30)=-.210,95 \% \mathrm{Cl}[-6.23,5.06], p=.835$ \\
\hline & Movement & & $\begin{array}{l}19.40(5.04)[11-29] \\
\text { BCa } 95 \% \text { Cl }[16.60,22.11]\end{array}$ & & $\begin{array}{l}21.45(7.13)[5-32] \\
\text { BCa } 95 \% \text { Cl }[18.14,24.37]\end{array}$ & $t(30)=.820,95 \% \mathrm{Cl}[-3.06,7.17], p=.419$ \\
\hline & Body position & & $\begin{array}{l}18.10(5.82)[8-27] \\
\text { BCa } 95 \% \text { Cl }[14.80,21.60]\end{array}$ & & $\begin{array}{l}18.86(5.85)[9-29] \\
\text { BCa } 95 \% \text { Cl }[16.27,21.55]\end{array}$ & $t(30)=.343,95 \% \mathrm{Cl}[-3.79,5.31], p=.734$ \\
\hline & Oral & & $\begin{array}{l}15.40(3.13)[13-21] \\
\text { BCa } 95 \% \text { Cl }[13.80,17.20]\end{array}$ & & $\begin{array}{l}16.95(4.51)[12-27] \\
\text { BCa } 95 \% \text { Cl }[15.31,18.78]\end{array}$ & $U=89.000, Z=-.860, p=.411, \eta^{2}=0.023$ \\
\hline & Seeking & & $\begin{array}{l}44.00(8.62)[28-55] \\
\text { BCa } 95 \% \text { Cl }[39.00,49.07]\end{array}$ & & $\begin{array}{l}45.18 \text { (12.51) [22-72] } \\
\text { BCa } 95 \% \mathrm{Cl}[40.08,50.58]\end{array}$ & $t(30)=.270,95 \% \mathrm{Cl}[-7.76,10.13), p=.789$ \\
\hline & Avoiding & & $\begin{array}{l}47.50(13.79)[23-71] \\
\text { BCa } 95 \% \mathrm{Cl}[39.30,55.70]\end{array}$ & & $\begin{array}{l}37.59 \text { (8.06) [19-51] } \\
\text { BCa 95\% Cl [33.95, 41.11] }\end{array}$ & $t(30)=-2.566,95 \% \mathrm{Cl}[-17.80,-2.02], p=.016$ \\
\hline
\end{tabular}


Table 2 ASD symptomatology by language level (Continued)

\begin{tabular}{|c|c|c|c|c|c|}
\hline & \multicolumn{2}{|c|}{ Verbal } & \multicolumn{2}{|c|}{ Minimally verbal ( $\geq 3 y$ o) } & \multirow[b]{2}{*}{ Comparison } \\
\hline & $n$ & Mean (SD) [range] & $n$ & Mean (SD) [range] & \\
\hline Sensitivity & & $\begin{array}{l}43.30(6.73)[32-54] \\
\text { BCa } 95 \% \text { Cl }[39.50,47.20]\end{array}$ & & $\begin{array}{l}41.36 \text { (9.67) }[26-65] \\
\text { BCa } 95 \% \text { Cl }[37.64,45.71]\end{array}$ & $t(30)=-.571,95 \% \mathrm{Cl}[-8.86,4.99], p=.572$ \\
\hline Registration & & $\begin{array}{l}54.40(13.01)[35-76] \\
\text { BCa } 95 \% \text { Cl }[46.90,61.93]\end{array}$ & & $\begin{array}{l}53.95(14.32)[33-78] \\
\text { BCa } 95 \% \mathrm{Cl}[47.72,60.51]\end{array}$ & $t(30)=-.084,95 \% \mathrm{Cl}[-11.30,10.41], p=.934$ \\
\hline Conduct & & $\begin{array}{l}21.70(4.60)[15-31] \\
\text { BCa } 95 \% \text { Cl }[19.30,24.20]\end{array}$ & & $\begin{array}{l}19.18 \text { (7.06) [9-30] } \\
\text { BCa 95\% Cl [16.29, 22.35] }\end{array}$ & $t(30)=-1.029,95 \% \mathrm{Cl}[-7.52,2.48], p=.312$ \\
\hline Social-emotional & & $\begin{array}{l}36.50(8.70)[22-50] \\
\text { BCa } 95 \% \text { Cl }[31.50,41.43]\end{array}$ & & $\begin{array}{l}27.82(7.23)[15-42] \\
\text { BCa } 95 \% \text { Cl }[24.80,30.68]\end{array}$ & $t(30)=-2.957,95 \% \mathrm{Cl}[-14.68,-2.69], p=.006$ \\
\hline Attentional & & $\begin{array}{l}28.60(7.43)[15-38] \\
\text { BCa } 95 \% \text { Cl }[24.20,32.70]\end{array}$ & & $\begin{array}{l}26.86 \text { (7.45) [15-42] } \\
\text { BCa 95\% Cl [24.11, 30.00] }\end{array}$ & $t(30)=-.612,95 \% \mathrm{Cl}[-7.53,4.06], p=.545$ \\
\hline
\end{tabular}

No correction for multiple comparisons. Only participants aged $\geq 3$ years old. ADOS-2: social affect (SA), repetitive and restrictive behaviours (RRB), calibrated severity score (CSS). CSS range 1-10 indicating increasing severity. ADI-R: Only participants aged 4 and up. Qualitative alterations of reciprocal social interactions (A), range 0-30; qualitative alterations of language/communication (B), non-verbal range 0-14, verbal range 0-26; restricted, repetitive and stereotyped behaviours and interests (C), range $0-12$. SRS (Social Responsiveness Scale), only verbal participants: total range 0-195, social awareness range 0-24; social cognition range 0-36, social communication range $0-66$, social motivation range $0-33$, autistic mannerisms range $0-36$. RBS-R (Repetitive Behavior Scale-Revised): stereotyped range 018 , self-injurious range $0-24$, compulsive range $0-24$, ritualistic range $0-18$, insistence in sameness range $0-33$, restricted range $0-12$. SP-2 (Sensory Profile-2): sensory sections: auditory range $0-40$, visual range $0-30$, touch range $0-55$, movement range $0-40$, body position range $0-40$ and oral range $0-50$; sensory quadrants: seeking range $0-95$, avoiding range $0-100$, sensitivity range $0-95$ and registration range $0-110$; behavioural section: conduct range $0-45$, socialemotional range $0-70$ and attentional range $0-50$. Confidence interval for the group means calculated with bootstrapping $(95 \% \mathrm{BCa} \mathrm{Cl})$. $95 \% \mathrm{Cl}$ for the mean difference calculated only for normally distributed variables

expressive V-scale scores and domain standard scores separating individuals by their language level are in Fig. 1.

In line with other findings [9], age seems to play a role in adaptive behaviour. Younger individuals showed higher standard scores, and age was inversely correlated with every VABS-3 domain (communication: $r_{s}(44)=-.296, p=.046$; daily living: $r(44)=-.512$, $p<.001$; socialization: $r(44)=-.421, p=.004 ;$ total adaptive: $r(44)=-.726, p=.001)$, a result compatible with the concept of "growing into deficit", i.e. increasing discrepancy with age-expected performance.

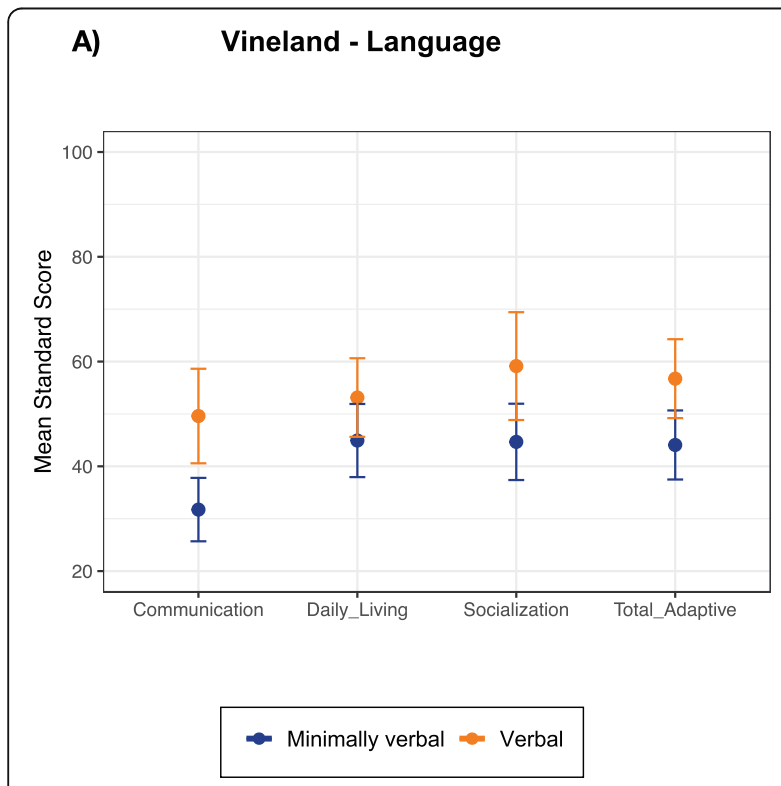

\section{B) Vineland Receptive vs. Expressive Subdomains}

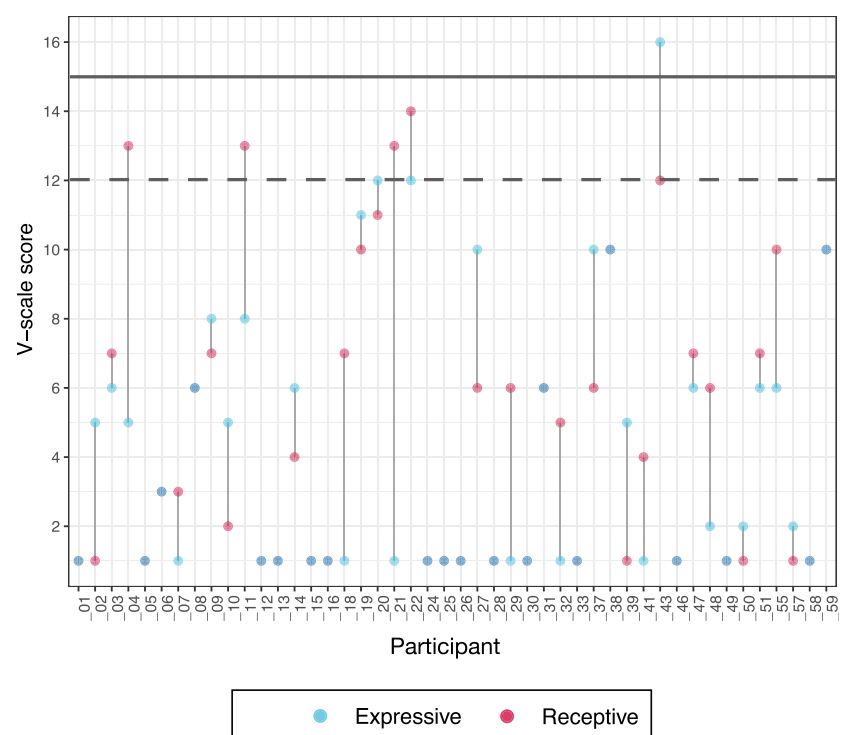

Fig. 1 VABS-3 standard scores by language level and receptive and expressive scores for every participant. A Differences based on language (only participants over age 3 considered): communication $(U=84.500, Z=-3.146, p=.002)$, daily living $(t(40)=-1.561,95 \% \mathrm{Cl}[-18.84,2.42], p=.126)$, socialization $(t(40)=-2.432,95 \% \mathrm{Cl}[-26.49,-2.44], p=.020)$, total adaptive $(t(40)=-2.510,95 \% \mathrm{Cl}[-22.85,-2.47], p=.016)$. "Verbal" $n=15 ;$; Minimally verbal" $n=27$. Error bars represent confidence interval of the mean. B Scores in receptive and expressive subdomains for each participant. Solid and dashed lines represent population mean and SD, respectively 


\section{Associated symptoms \\ Behavioural alterations}

Aggressive behaviours were present in $23.7 \%$ of the sample as measured by the CBCL. The ADI-R also recorded some degree of aggressive behaviour in $35.8 \%$ of individuals across lifetime, while $52.8 \%$ had self-injurious behaviours. The CBCL and SDQ captured clinically relevant scores for hyperactivity, attentional problems and difficulties relating to peers and establishing social contact and appear in Fig. 2 as percentage of individuals complying with borderline/clinical scores. In SDQ, verbal participants showed more prosocial behaviour than non-verbal individuals (5.40 vs. $2.87 ; U=53.500, Z=$ $-2.443, p=.015$, Hedges' $g=0.984$ ), whereas there were no apparent differences in respect to language in the CBCL. Within the ABC-C, the largest differences between verbal and $>3$ years old, non-verbal participants were found for stereotyped behaviour and repetitive speech. Scores can be found in Supplementary table 2.

\section{Medical comorbidities}

The most widespread conditions, reported in 53 cases, were musculoskeletal, especially hypotonia and bone anomalies such as claw feet and kyphosis. Recurrent respiratory tract, ear or urinary tract infections were reported in 45 cases, and usually struck hardest during the first 4 years of life, although they persisted in some cases. Two individuals suffered meningitis within 4 months postnatally. Thirty-five participants suffered from lower gastrointestinal disturbances (mainly constipation/diarrhoea) and 30 from gastroesophageal reflux. Thirty-three had neurologic findings of varying relevance, of which 7 had epilepsy and 6 a history of febrile seizures or epileptic-like episodes. Respiratory problems

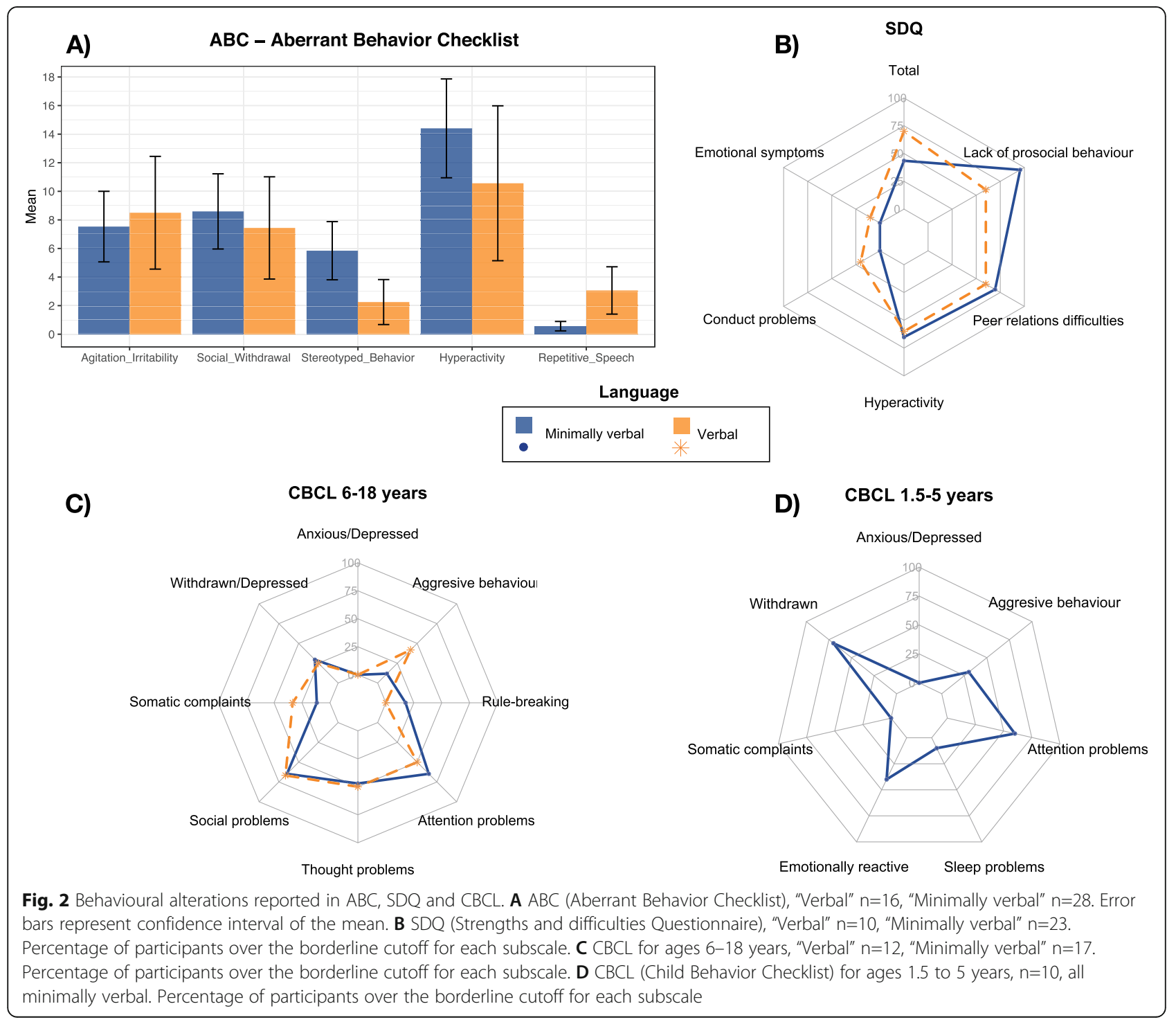


were common among 30 participants. Twenty-four individuals showed food intolerances, especially to lactose and legumes, and 5 had food allergies. Important sleep difficulties appeared in 19 individuals, including difficulty falling asleep, recurrent waking up or short sleeping times. Eighteen patients had various renal anomalies, including asymmetric or duplex kidneys, and 11 had genitourinary tract complaints, such as recurrent infections, urethral reflux or hypospadias. Sixteen participants had skin complaints, the most common being atopic dermatitis. Fifteen participants reported low iron levels. Fourteen had cardiac anomalies. Ten had endocrinemetabolic problems. Supplementary tables 3 and 4 summarise the reported medical comorbidities using CIRS items of any severity and further medical history. Results are retrospective and encompass events occurring at any point during the participants' lifetime.

\section{Genetic information and cluster analysis}

Genetic information including deletion size was available for 53 participants (52 aCGH and 1 MLPA with precise estimated loss); another exome sequencing test reported a point mutation affecting SHANK3. The remaining 6 lacked deletion size information due to the type of test (1 karyotype, 2 FISH, 3 MLPA). The reported information also identified 4 ring chromosomes, 6 mosaics ranging from 35 to $70 \%$ and 15 individuals with additional CNVs.

We tested the association of deletion size with language, motor milestones, regression and ADOS-2 CSS score (Fig. 3). Participants with delayed motor milestone acquisition (i.e. delayed walking) apparently had larger deletion sizes than those who walked at a typical age $(t(49)=-6.147,95 \%$ CI $[-4.25,-2.16], p<.001)$, and so did participants with absence of language compared to those with fluid speech $(F(3,49)=3.012, p=.039)$. Detailed information on deletion size and its association with phenotypic variables are presented in Supplementary table 5.

To explore similarities within the phenotype, we conducted an exploratory hierarchical cluster analysis on 42 participants using clinical and demographic data. This resulted in four groups for which the clearest separation was the deletion size together with ADOS-2 CSS scores. Cluster 1 was composed of 11 participants with deletions of medium-to-large size $(\mathrm{M}=4.16 \mathrm{Mb}(1.70))$ and low CSS scores (median $=3$, range $=[2-4]$ ). Cluster 2 had 6 participants with relatively large deletions $(\mathrm{M}=$ $5.51 \mathrm{Mb}(1.47)$ ) and high CSS scores (median $=8.5$, range $=$ [6-10] ). Cluster 3 was formed by 10 individuals with mean deletion size of $2.60 \mathrm{Mb}(.96)$ and accompanying autistic symptoms (median $=6$, range $=[6-7]$ ) and cluster 4 gathered subjects with the smaller deletions $(\mathrm{M}=$ $.35 \mathrm{Mb}(.46))$, varying autistic symptom severity (median= 6 , range $=[3-9])$, and the lowest proportion of participants with delayed motor milestones. Distribution of clinical variables and sex within clusters is shown in Supplementary Figure 1 and Supplementary table 6.

\section{Discussion}

This is the first general phenotypic description of a representative cohort of Spanish PMS patients. We evaluated sixty patients $(85 \%$ of them children and adolescents) with an even sex distribution. Participants' independent walking and language acquisition were delayed. Forty-two percent of participants experienced development regression or loss of skills, with half of them recovering skills over varying periods. Adaptive functioning ranged from very to moderately low. Autistic symptomatology was variable in type and severity, with a median ADOS-2 CSS of 6 for every domain. Caregivers often reported that participants experienced sensory problems and attention and hyperactivity difficulties, as well as medical comorbidities, such as gastrointestinal issues, respiratory infections, neurological anomalies and sleep difficulties.

\section{Developmental milestones}

Our findings align with previous descriptions of patients with PMS. With regards to developmental milestones, our data suggest important motor delay in up to $90 \%$ of the sample and generalized language impairment, with $14.8 \%$ of participants using fluid speech, comparable with other studies $[3,4,14,37]$. There were no apparent differences between receptive vs. expressive communicative skills, although results showed wide variability both within and between subjects.

\section{Adaptive skills, ASD and regression}

Per the VABS-3, all participants functioned below their chronological age, as in previous studies [14, 24], with scores two or more SD below average, suggesting that individuals with PMS, regardless of autistic symptomatology, may be in the moderate to severe range of ID. Adaptive behaviour is necessary to determine the level of ID, and ID status can be granted on the basis of a delayed adaptive level in relation to chronological expectations. Low adaptive skills are therefore part of the mental health classification systems (i.e. DSM and CIE) and a good index to determine the presence of ID [29].

However, less affected individuals with PMS may go undetected because genetic testing is likelier for individuals with low IQ or dysmorphic features. Despite the generalised difficulties in the communication domain, our group results are mostly in line with the patterns observed in ID, with higher socialization scores. A more exhaustive profile of adaptive skills taking into account relevant variables, such as age, degree of ID or autistic symptoms, is warranted. 


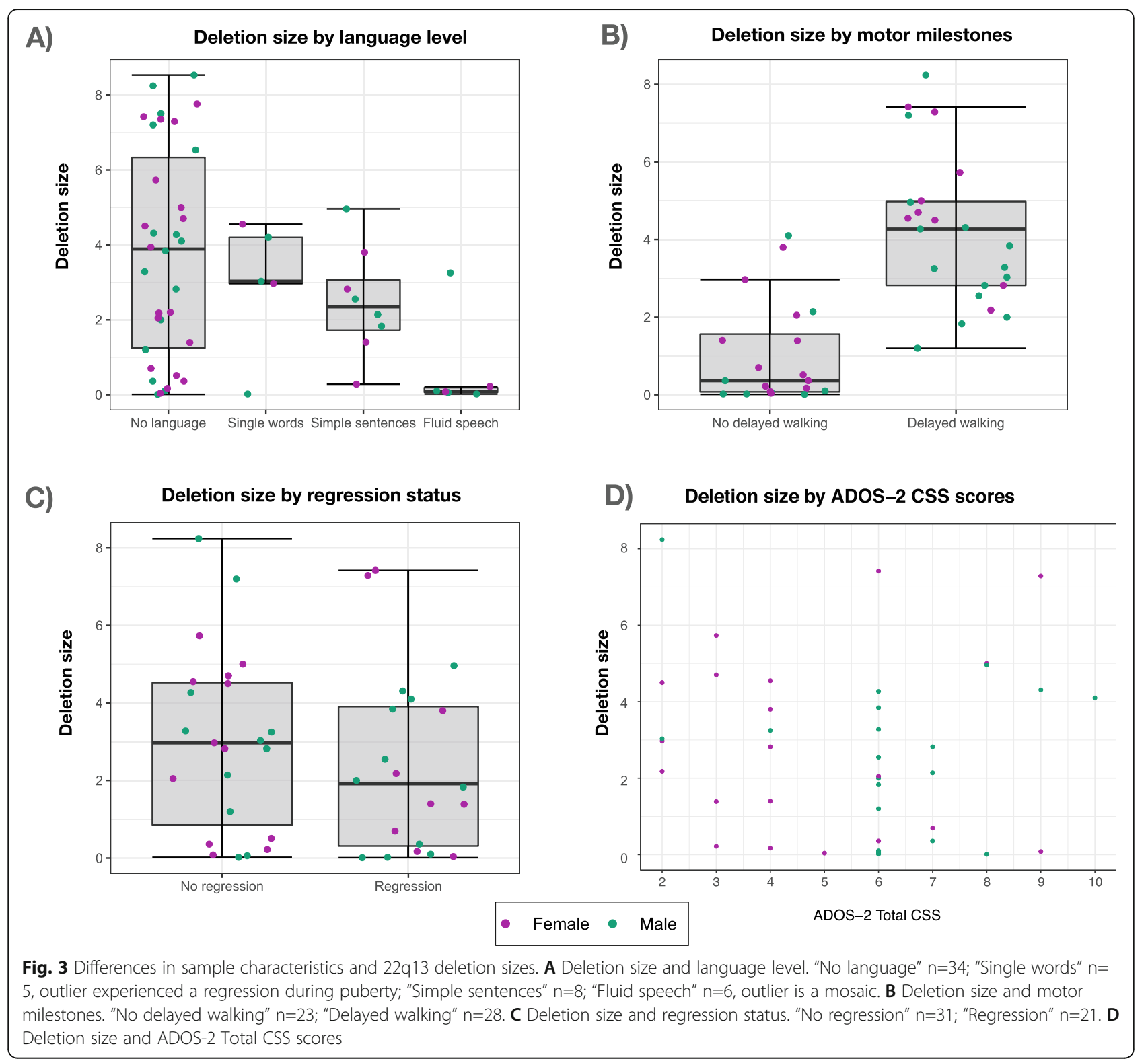

The presence of autistic symptoms in PMS is largely acknowledged; nevertheless, the estimated prevalence of ASD in PMS remains disputed, with the highest figures pointing towards more than $80 \%$ [14], but with a broad range, that likely depends on the diagnostic tools used. Some authors have suggested that PMS may have a different autistic-like profile than strictly described by DSM-IV $[14,38]$ or even posit that the characteristics of PMS may be related to communication impairments rather than an autistic manifestation [39]. Our results show a range of behaviours compatible with ASD, including sociocommunicative difficulties, resistance to change, unusual or restricted interests and stereotyped and ritualistic behaviour. Interestingly, verbal participants showed higher scores in subscales measuring ritualized behaviours, more connected to insistence in sameness (IS) than repetitive sensorimotor actions (RSM) within RRBs, and might be associated with less social impairment, while RSM appear more strongly associated with cognitive ability [40]. Sensory processing anomalies were also widespread, especially those related to tactile and proprioceptive stimuli. Nevertheless, these results must be interpreted with care, as even goldstandard instruments show suboptimal validity in populations with profound ID [41]. Categorical assessment in such populations is challenging, but the inclusion of clinical judgement would likely refine these estimates and more accurately inform about the prevalence of autism in a sample like this. While it was not the intention of this work to provide a clinical diagnosis of autism, we 
understand that this may be necessary for families in particular contexts to be granted services.

Researchers should integrate robust standardised tools, direct observation and clinical evaluation by experienced professionals to explore and describe more deeply the ASD profile in PMS. This would help account for potential differences with idiopathic autism or other forms of syndromic autism and discern whether these manifestations correspond to a distinct ASD profile.

Concerning developmental regression, we identified that half of the $42 \%$ of participants that had an apparent loss of abilities regained previous functioning. The percentage of participants with permanent deficits $(22 \%)$ we found is in line with previous findings of $26-28 \%[7,14]$. The most affected areas for our sample were communication/language and social engagement, while other areas, such as self-help or motor skills, were not reported as often as in previous studies. Nevertheless, the assessment tools may not detect regression in children with very low abilities. Some patients experienced a very specific global regression during early puberty or adolescence, an interesting finding worth follow-up investigation.

\section{Clinical implications of behavioural problems and medical comorbidities}

As in previous studies, hyperactivity and attention deficit were prevalent across all tools [3, 14, 42]. Although these symptoms are commonly observed in ID and may not be specific to PMS, they merit attention. Aggression and self-injurious behaviour were problematic for one third and half of the sample, respectively, so interventions aimed at improving not only communication, but also self-regulation strategies are desirable. Clinically relevant social withdrawal and internalising emotional symptoms were rare, as reported by parent questionnaires, which highlights the difficulty of evaluating internalising symptomatology in this population and a need for appropriate tools. However, mood and behavioural symptoms and psychiatric decompensation have been previously reported and systematically reviewed [43]. This exposes the importance of accurate and prompt assessment of changes in the clinical course, including mood lability and hypo- or hyperthymic profiles.

Similar to previous work [44], the most common medical comorbidities included gastrointestinal disturbances and gastroesophageal reflux, respiratory infections, food intolerances, sleep disturbances, skin complaints, genitourinary or renal anomalies, and epilepsy or febrile seizures, along with recurrent paediatric infections. It is of utmost importance to monitor the health of these patients, particularly in the context of their difficulties communicating pain or distress. Health status impacts behaviour, sleep and well-being in general, and therefore developmental and educational opportunities. Health care professionals need training in recognising and diagnosing medical comorbidities in patients with severe ID.

\section{Genotype-phenotype associations and hierarchical clustering}

Regarding phenotype association with deletion size, we found mixed results across several traits. For example, motor development and language were less affected in individuals with smaller deletions, and we found no association with developmental regression or epilepsy, as previously reported $[7,20]$. There was no clear association with ADOS or ADI-R or other behavioural manifestations, such as aggression or hyperactivity [4]. Nevertheless, our study included only reported information stemming from different genetic tests and resolution levels. A deeper analysis resulting from more precise genetic testing could help identify therapeutic targets and elucidate the relationship between these patients' development and specific clinical outcomes and their genomes.

Our cluster analysis yielded four groups on the basis of deletion size and autistic symptom severity. While certain selected characteristics were apparently grouped in a congruent fashion with previous findings (e.g. motor delay), others were not (e.g. language status), which is surprising considering that deletion size was associated with language ability. More fine-grained analysis of clinical characteristics and their relation to deletion size is warranted.

\section{Limitations and future implications}

First, our sample may encompass only an extreme subset of the PMS phenotype, which are the ones likeliest to have been diagnosed so far (younger and more affected individuals), and therefore may suffer from ascertainment bias. The profile may change in a couple of years when more systematic genetic testing for individuals with developmental delays exists. Future efforts should be put on harmonising measures across countries to build a common database for understanding this unique condition. Second, collecting all the questionnaires and other variables from the total sample proved to be difficult, due to time constraints and incomplete compliance with "off-site" measures. Further caveats are the lack of a standardised measure of cognitive ability (which also limits the interpretation of the results concerning verbal status), due to the difficulty of rigorously assessing a sample with a wide range of ages, severity of ID and limited language comprehension, and the lack of appropriate assessment tools, which hampers a more precise account of the syndrome herein discussed. To this effect, efforts are being made to review and discuss available instruments that may prove effective [45], since 
traditionally used methods are generally validated with normative samples. ADOS and ADI-R, for example, recognise as main limitations the assessment of autistic symptoms in ID or very young patients [23, 28, 46], and we acknowledge that the inclusion of clinical judgement regarding ASD symptoms would have provided a contextualized insight into the diagnostic prevalence of autism. However, the wide range of behavioural, clinical and genetic information we included depicts a clear picture of our sample and offers an unprecedented broad profile of a representative Spanish PMS cohort.

\section{Conclusions}

This study adds to the available information on the clinical manifestation of PMS patients, who typically show impaired adaptive skills, speech acquisition delay or absence of language, and intellectual disability of variable degree, along with a number of medical and behavioural challenges, such as ASD, developmental regression and hyperactivity, some of which appear to correlate with deletion size. We emphasise the need for specific measurement tools that overcome current limitations and the importance of accurate psychiatric assessment to identify possible emotional dysregulations. Finding appropriate psychotherapeutic approaches and improving pharmacological treatments is crucial, as is understanding the response of this population to available medications and ascertaining whether they could benefit from specific and individualised treatments now under development. This will allow for personalised pharmacological and psychotherapeutic approaches. We highlight the need for longitudinal studies, to better describe PMS developmental trajectories and clinical courses, and better predict potential comorbidities of patients with PhelanMcDermid syndrome.

\section{Abbreviations \\ PMS: Phelan-McDermid syndrome; ASD: Autism spectrum disorder; ID: Intellectual disability; M: Mean; SD: Standard deviation; ADOS-2: Autism Diagnostic Observation Schedule-2; ADI-R: Autism Diagnostic Interview- Revised; RBS-R: Repetitive Behaviours Scale-Revised; SRS: Social Responsiveness Scale; SP-2: Winnie Dunn Sensory Profile-2; CSS: Calibrated severity scores; VABS-3: Vineland Adaptive Behavior Scales-3; ABC-C: Aberrant Behavior Checklist-Community; CBCL: Child Behavior Checklist; \\ SDQ: Strengths and Difficulties Questionnaire; CIRS: Cumulative IIIness Rating Scale; FISH: Fluorescent in situ hybridization; MLPA: Multiplex ligation probe amplification; array-CGH/aCGH: Microarray-based comparative genome hybridization}

\section{Supplementary Information}

The online version contains supplementary material available at https://doi. org/10.1186/s11689-021-09370-5.

Additional file 1:. Supplementary Figure 1. Hierarchical clustering dendrogram showing four clusters with the distribution of the included variables. Supplementary table 1 . Sensory profile anomalies divided by language level. Supplementary table 2. Scores in behavioural questionnaires based on language level. Supplementary table 3 Medical complaints (parent interviews and medical records). Supplementary table 4. CIRS classification of reported events. Supplementary table 5. Differences in sample characteristics and 22q13 deletion size and trait correlation with deletion size. Supplementary table 6. Cluster number and clinical characteristics.

\section{Acknowledgements}

We are grateful to Asociación Phelan-McDermid España for their support, and we thank all participants and their families for their collaboration. We thank Lucas Laursen for the English language editing.

\section{Authors' contributions}

$\mathrm{MBO}$ and MPR designed the study and wrote the manuscript. MBO intermediated the communication with families and carried out the assessments, data analysis and interpretation. AGA carried out several assessments, and AGA and ASJC were major contributors to the manuscript. JGP offered support involving the genetic information of participants, statistical analysis and figure generation. PHJ and MPR offered clinical insight on the psychiatric aspects of the syndrome. The authors read and approved the final manuscript.

\section{Funding}

This work was supported by the Spanish Ministry of Science, Innovation and Universities, Instituto de Salud Carlos III (FIS PI17/00819), co-financed by ERDF Funds from the European Commission, "A way of making Europe", CIBERSAM Madrid Regional Government (B2017/BMD-3740 AGES-CM-2), European Union Structural Funds, European Union Seventh Framework Program, European Union H2020 Program under the Innovative Medicines Initiative 2 Joint Undertaking (grant agreement No 115916, Project PRISM and grant agreement No 777394, Project AIMS-2-TRIALS), Fundación Familia Alonso, Fundación Alicia Koplowitz and Fundación Mutua Madrileña. Asociación Síndrome Phelan-McDermid España funded the collection and analysis of data, as well as the publication of this article. MBO has been a recipient of a pre-doctoral fellowship (PFIS: Contratos Predoctorales de Formación en Investigación en Salud) from the Spanish Ministry of Science, Innovation and Universities through Instituto de Salud Carlos III (FI18/00233), and this article is part of her PhD studies.

\section{Availability of data and materials \\ The datasets used and/or analysed during the current study are available from the corresponding author on reasonable request.}

\section{Declarations}

Ethics approval and consent to participate

Caregivers gave informed consent in all cases (all adult participants were legally incapacitated). The ethics committee of the Hospital General Universitario Gregorio Marañón approved the study.

\section{Consent for publication}

Not applicable.

\section{Competing interests}

AGA, JGP and PHJ declare that they have no competing interests. MBO received salary for the study coordination and data collection from Asociación Síndrome Phelan-McDermid España. ASJC has been a consultant for F. Hoffmann-La Roche Ltd, consults for Servier and is involved in clinical trials conducted by Servier. The present work is unrelated to the above grants and relationships. MPR has been a consultant for Fundación Alicia Koplowitz, the EMA and Instituto de Salud Carlos III (ISCIII); consults for Servier and Exeltis; has received grant/research support from CIBERSAM, Fundación Alicia Koplowitz, Fundación Mutua Madrileña, ISCIII and Horizon2020; receives travel support from Exeltis; and is involved in clinical trials conducted by Servier, F. Hoffmann-La Roche Ltd and Horizon2020. The present work is unrelated to the above grants and relationships.

\section{Author details}

'Department of Child and Adolescent Psychiatry, Institute of Psychiatry and Mental Health, Hospital General Universitario Gregorio Marañón, Calle Ibiza 43, 28009 Madrid, Spain. ${ }^{2}$ liSGM, Instituto de Investigación Sanitaria Gregorio 
Marañón, Madrid, Spain. ${ }^{3}$ School of Psychology, Universidad Autónoma, Madrid, Spain. ${ }^{4}$ School of Medicine, Universidad Complutense, Madrid, Spain. ${ }^{5}$ CIBERSAM, Centro de Investigación Biomédica en Red Salud Mental, Madrid, Spain.

\section{Received: 23 June 2020 Accepted: 28 May 2021}

Published online: 10 July 2021

\section{References}

1. Phelan MC, Thomas GR, Saul RA, Rogers RC, Taylor HA, Wenger DA, et al Cytogenetic, biochemical, and molecular analyses of a 22 q13 deletion. Am J Med Genet. 1992;43(5):872-6. https://doi.org/10.1002/ajmg.1320430524.

2. Phelan K, McDermid H. The 22q13. 3 deletion syndrome (Phelan-McDermid syndrome). Molecular syndromology. 2011;2(3-5):186-201. https://doi.org/1 $0.1159 / 000334260$.

3. De Rubeis S, Siper PM, Durkin A, Weissman J, Muratet F, Halpern D, et al. Delineation of the genetic and clinical spectrum of Phelan-McDermid syndrome caused by SHANK3 point mutations. Molecular autism. 2018;9(1): 31. https://doi.org/10.1186/s13229-018-0205-9.

4. Sarasua SM, Boccuto L, Sharp JL, Dwivedi A, Chen C-F, Rollins JD, et al. Clinical and genomic evaluation of 201 patients with Phelan-McDermid syndrome. Hum Genet. 2014;133(7):847-59. https://doi.org/10.1007/s00439014-1423-7.

5. Yitzchak Frank M, Jamison JM, Tavassoli T, Kolevzon M. A prospective study of neurological abnormalities in Phelan-McDermid syndrome. Journal of Rare Disorders. 2017;5(1):1.

6. Denayer A, Van Esch H, De Ravel T, Frijns J-P, Van Buggenhout G, Vogels A, et al. Neuropsychopathology in 7 patients with the 22q13 deletion syndrome: presence of bipolar disorder and progressive loss of skills. Molecular syndromology. 2012;3(1):14-20. https://doi.org/10.1159/00033911 9

7. Reierson G, Bernstein J, Froehlich-Santino W, Urban A, Purmann C, Berquist $S$, et al. Characterizing regression in Phelan McDermid Syndrome (22q13 deletion syndrome). J Psychiatr Res. 2017;91:139-44. https://doi.org/10.1016/ j.jpsychires.2017.03.010

8. Betancur C, Buxbaum JD. SHANK3 haploinsufficiency: a "common" but underdiagnosed highly penetrant monogenic cause of autism spectrum disorders: BioMed Central; 2013.

9. Zwanenburg RJ, Ruiter SA, van den Heuvel ER, Flapper BC, Van RavenswaaijArts CM. Developmental phenotype in Phelan-McDermid (22q13. 3 deletion) syndrome: a systematic and prospective study in 34 children. $J$ Neurodev Disord. 2016;8(1):16.

10. Guilmatre A, Huguet G, Delorme R, Bourgeron T. The emerging role of SHANK genes in neuropsychiatric disorders. Developmental neurobiology. 2014;74(2):113-22. https://doi.org/10.1002/dneu.22128.

11. Leblond CS, Nava C, Polge A, Gauthier J, Huguet G, Lumbroso S, et al. Metaanalysis of SHANK mutations in autism spectrum disorders: a gradient of severity in cognitive impairments. PLoS Genet. 2014;10(9):e1004580. https:// doi.org/10.1371/journal.pgen.1004580.

12. Bourgeron T. A synaptic trek to autism. Curr Opin Neurobiol. 2009:19(2):2314. https://doi.org/10.1016/j.conb.2009.06.003

13. Wilson H, Wong A, Shaw S, Tse W, Stapleton G, Phelan M, et al. Molecular characterisation of the 22q13 deletion syndrome supports the role of haploinsufficiency of SHANK3/PROSAP2 in the major neurological symptoms. J Med Genet. 2003;40(8):575-84. https://doi.org/10.1136/jmg.40. 8.575

14. Soorya L, Kolevzon A, Zweifach J, Lim T, Dobry Y, Schwartz L, et al. Prospective investigation of autism and genotype-phenotype correlations in 22q13 deletion syndrome and SHANK3 deficiency. Molecular autism. 2013; 4(1):18. https://doi.org/10.1186/2040-2392-4-18

15. Cooper GM, Coe BP, Girirajan S, Rosenfeld JA, Vu TH, Baker C, et al. A copy number variation morbidity map of developmental delay. Nat Genet. 2011 43(9):838-46. https://doi.org/10.1038/ng.909.

16. Gong $X$, Jiang $Y-W$, Zhang $X, A n Y$, Zhang J, Wu Y, et al. High proportion of $22 q 13$ deletions and SHANK3 mutations in Chinese patients with intellectual disability. PLoS One. 2012;7(4):e34739.

17. Tabet A-C, Rolland T, Ducloy M, Lévy J, Buratti J, Mathieu A, et al. A framework to identify contributing genes in patients with Phelan-McDermid syndrome. NPJ genomic medicine. 2017:2(1):32. https://doi.org/10.1038/s41 525-017-0035-2
18. Sarasua SM, Dwivedi A, Boccuto L, Rollins JD, Chen C-F, Rogers RC, et al. Association between deletion size and important phenotypes expands the genomic region of interest in Phelan-McDermid syndrome (22q13 deletion syndrome). J Med Genet. 2011;48(11):761-6. https://doi.org/10.1136/ jmedgenet-2011-100225.

19. Oberman LM, Boccuto L, Cascio L, Sarasua S, Kaufmann WE. Autism spectrum disorder in Phelan-McDermid syndrome: initial characterization and genotype-phenotype correlations. Orphanet journal of rare diseases. 2015;10(1):105. https://doi.org/10.1186/s13023-015-0323-9.

20. Sarasua SM, Dwivedi A, Boccuto L, Chen C-F, Sharp JL, Rollins JD, et al. 22q13. 2q13. 32 genomic regions associated with severity of speech delay, developmental delay, and physical features in Phelan-McDermid syndrome. Genetics in Medicine. 2014;16(4):318.

21. Parellada M, Boada L, Moreno C, Llorente C, Romo J, Muela C, et al. Specialty care programme for autism spectrum disorders in an urban population: a case-management model for health care delivery in an ASD population. European Psychiatry. 2013;28(2):102-9. https://doi.org/10.1016/j. eurpsy.2011.06.004.

22. Lord C, Rutter M, DiLavore P, Risi S, Gotham K, Bishop S, et al. ADOS-2. Escala de Observación para el Diagnóstico del Autismo-2 Manual (T Luque, adaptadora). Madrid: TEA Ediciones; 2015.

23. Rutter M, Le Couteur A, Lord C. ADI-R: Entrevista para el diagnóstico del autismo-revisada: TEA; 2006.

24. Phelan MC, Rogers RC, Saul RA, Stapleton GA, Sweet $K$, McDermid H, et al. 22q13 deletion syndrome. Am J Med Genet. 2001:101(2):91-9. https://doi. org/10.1002/1096-8628(20010615)101:2\%3C91::AID-AJMG1340\%3E3.0.CO;2-C.

25. Constantino JN, Davis SA, Todd RD, Schindler MK, Gross MM, Brophy SL, et al. Validation of a brief quantitative measure of autistic traits: comparison of the social responsiveness scale with the autism diagnostic interviewrevised. J Autism Dev Disord. 2003;33(4):427-33. https://doi.org/10.1023/A:1 025014929212.

26. Dunn W. Sensory profile 2: Psych Corporation; 2014.

27. Gotham K, Pickles A, Lord C. Standardizing ADOS scores for a measure of severity in autism spectrum disorders. J Autism Dev Disord. 2009:39(5):693705. https://doi.org/10.1007/s10803-008-0674-3.

28. Sappok T, Diefenbacher A, Budczies J, Schade C, Grubich C, Bergmann T, et al. Diagnosing autism in a clinical sample of adults with intellectual disabilities: how useful are the ADOS and the ADI-R? Res Dev Disabil. 2013; 34(5):1642-55. https://doi.org/10.1016/j.ridd.2013.01.028.

29. Sparrow S, Cicchetti DV, Saulnier CA. Vineland adaptive behavior scales, (Vineland-3). Antonio: Psychological Corporation; 2016.

30. Aman MG, Burrow WH, Wolford PL. The Aberrant Behavior ChecklistCommunity: factor validity and effect of subject variables for adults in group homes. Am J Ment Retard. 1995.

31. Achenbach T, Rescorla L. Manual for the ASEBA school-age forms \& profiles: an integrated system of multi-informant assessment Burlington, $\mathrm{VT}$ : University of Vermont. Research Center for Children, Youth, \& Families. 2001; 1617.

32. Goodman R. The Strengths and Difficulties Questionnaire: a research note. J Child Psychol Psychiatry. 1997;38(5):581-6. https://doi.org/10.1111/j.1469-761 0.1997.tb01545.x.

33. Linn BS, LINN MW, Gurel L. Cumulative illness rating scale. J Am Geriatr Soc. 1968;16(5):622-6. https://doi.org/10.1111/j.1532-5415.1968.tb02103.x.

34. Harris PA, Taylor R, Thielke R, Payne J, Gonzalez N, Conde JG. Research electronic data capture (REDCap) — a metadata-driven methodology and workflow process for providing translational research informatics support. J Biomed Inform. 2009:42(2):377-81. https://doi.org/10.1016/j.jbi.2008.08.010.

35. SPSS I. PASW statistics 18. Chicago: SPSS Inc.; 2009.

36. R Core Team. R: a language and environment for statistical computing. Vienna, Austria: R Foundation for Statistical Computing; 2020.

37. Samogy-Costa Cl, Varella-Branco E, Monfardini F, Ferraz H, Fock RA, Barbosa RHA, et al. A Brazilian cohort of individuals with Phelan-McDermid syndrome: genotype-phenotype correlation and identification of an atypical case. J Neurodev Disord. 2019;11(1):13. https://doi.org/10.1186/s11689-0199273-1.

38. Philippe A, Boddaert N, Vaivre-Douret L, Robel L, Danon-Boileau L, Malan V, et al. Neurobehavioral profile and brain imaging study of the 22q13. 3 deletion syndrome in childhood. Pediatrics. 2008;122(2):e376-e82. https:// doi.org/10.1542/peds.2007-2584.

39. Ponson L, Gomot M, Blanc R, Barthelemy C, Roux S, Munnich A, et al. 22q13 deletion syndrome: communication disorder or autism? Evidence from a 
specific clinical and neurophysiological phenotype. Transl Psychiatry. 2018; 8(1):146. https://doi.org/10.1038/s41398-018-0212-9.

40. Richler J, Huerta M, Bishop SL, Lord C. Developmental trajectories of restricted and repetitive behaviors and interests in children with autism spectrum disorders. Dev Psychopathol. 2010;22(1):55-69. https://doi.org/10.1 017/S0954579409990265.

41. Thurm A, Farmer C, Salzman E, Lord C, Bishop S. State of the field: Differentiating intellectual disability from autism spectrum disorder. Frontiers in psychiatry. 2019;10. https://doi.org/10.3389/fpsyt.2019.00526.

42. Egger J, Zwanenburg R, van Ravenswaaij-Arts C, Kleefstra T, Verhoeven W. Neuropsychological phenotype and psychopathology in seven adult patients with Phelan-McDermid syndrome: implications for treatment strategy. Genes Brain Behav. 2016;15(4):395-404. https://doi.org/10.1111/ gbb.12285.

43. Kolevzon A, Delaby E, Berry-Kravis E, Buxbaum JD, Betancur C. Neuropsychiatric decompensation in adolescents and adults with PhelanMcDermid syndrome: a systematic review of the literature. Molecular Autism. 2019;10(1):1-22.

44. Kolevzon A, Angarita B, Bush L, Wang AT, Frank Y, Yang A, et al. PhelanMcDermid syndrome: a review of the literature and practice parameters for medical assessment and monitoring. J Neurodev Disord. 2014;6(1):39. https://doi.org/10.1186/1866-1955-6-39.

45. Soorya L, Leon J, Trelles MP, Thurm A. Framework for assessing individuals with rare genetic disorders associated with profound intellectual and multiple disabilities (PIMD): the example of Phelan McDermid syndrome. Clin Neuropsychol. 2018;32(7):1226-55. https://doi.org/10.1080/13854046.201 7.1413211

46. Gotham K, Risi S, Pickles A, Lord C. The Autism Diagnostic Observation Schedule: revised algorithms for improved diagnostic validity. J Autism Dev Disord. 2007;37(4):613-27. https://doi.org/10.1007/s10803-006-0280-1.

\section{Publisher's Note}

Springer Nature remains neutral with regard to jurisdictional claims in published maps and institutional affiliations.

Ready to submit your research? Choose BMC and benefit from:

- fast, convenient online submission

- thorough peer review by experienced researchers in your field

- rapid publication on acceptance

- support for research data, including large and complex data types

- gold Open Access which fosters wider collaboration and increased citations

- maximum visibility for your research: over $100 \mathrm{M}$ website views per year

At $\mathrm{BMC}$, research is always in progress.

Learn more biomedcentral.com/submissions 